\title{
Cone geometry optimization and thermal behavior for lithium-ion battery separators
}

Cite as: AIP Conference Proceedings 2293, 260006 (2020); https://doi.org/10.1063/5.0026453 Published Online: 25 November 2020

D. Miranda, R. Gonçalves, F. Miranda, E. Vilhena, S. Lanceros-Méndez, and C. M. Costa

\section{ARTICLES YOU MAY BE INTERESTED IN}

Theoretical simulation of different 3D separator geometries for lithium-ion batteries AIP Conference Proceedings 2293, 260005 (2020); https://doi.org/10.1063/5.0026447

Preface of the "5th Symposium on Modelling and Simulation in Computer Sciences and Engineering"

AIP Conference Proceedings 2293, 260001 (2020); https://doi.org/10.1063/5.0026557

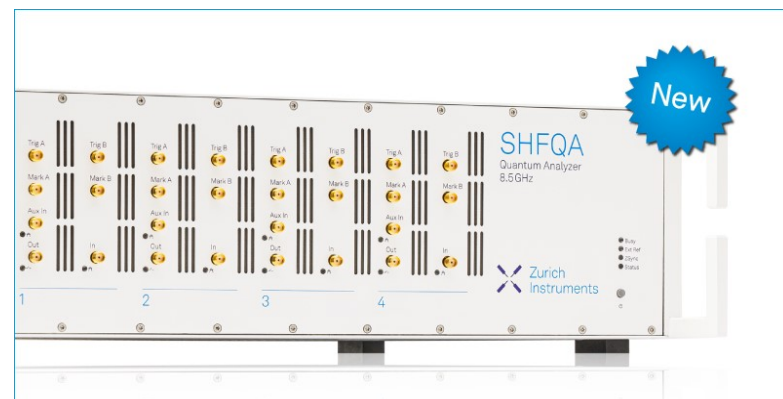

\section{Your Qubits. Measured.}

Meet the next generation of quantum analyzers

- Readout for up to 64 qubits

- Operation at up to $8.5 \mathrm{GHz}$.

- Signal optimization with minimal latency 


\title{
Cone Geometry Optimization and Thermal Behavior for Lithium-Ion Battery Separators
}

\author{
D. Miranda ${ }^{1, a)}$, R. Gonçalves ${ }^{2, b)}$, F. Miranda ${ }^{3,4, c)}$, E. Vilhena ${ }^{1, d)}$, S. Lanceros- \\ Méndez ${ }^{5,6, \mathrm{e}}$ and C. M. Costa ${ }^{2,7, \mathrm{f})}$ \\ 12Ai- Polytechnic Institute of Cávado and Ave, Barcelos, Portugal \\ ${ }^{2}$ Centro de Quimica, Universidade do Minho, 4710-057 Braga, Portugal \\ ${ }^{3}$ CIDMA, Universidade de Aveiro, Aveiro, Portugal \\ ${ }_{4}^{4}$ Instituto Politécnico de Viana do Castelo, Viana do Castelo, Portugal \\ ${ }^{5}$ BCMaterials, Basque Center for Materials, Applications and Nanostructures, UPV/EHU Science Park, 48940 \\ Leioa, Spain \\ ${ }^{6} I K E R B A S Q U E$, Basque Foundation for Science, 48013 Bilbao, Spain \\ ${ }^{7}$ Centro de Física, Universidade do Minho, 4710-057 Braga, Portugal \\ a)Corresponding author: damiranda@ipca.pt \\ b) r.goncalves@quimica.uminho.pt \\ c)fmiranda@estg.ipvc.pt \\ d) evilhena@ipca.pt \\ e)senentxu.lanceros@bcmaterials.net \\ f)cmscosta@fisica.uminho.pt
}

\begin{abstract}
A 3D cone separator geometry for lithium-ion batteries has been optimized taking into account the increase of radius size of one side. Theoretical simulations have been carried out for evaluating the influence of radius size in the cone structure at different discharge rates $(1 \mathrm{C}$ and $60 \mathrm{C})$ in which it was also determined the produced ohmic heat. The value of the discharge capacity in the cone structure depends on the increases of the radius, which is correlated with the electrolyte volume and interface between free electrolyte/cathode. The optimum balance of these parameters is essential for obtaining higher battery performance through this geometry that can be used in the next generation of lithium-ion batteries.
\end{abstract}

Keywords: Separator, simulation, capacity, lithium ion battery.

PACS: 88.80.F-, 88.80.ff, 88.05.-b

\section{INTRODUCTION}

In a highly technological society an increasing energy demand is verified, which must be accompanied by a reduction of fossil fuels dependence. Further, an improvement is essential in electrochemical energy storage devices, including increasing energy density in batteries (lithium-ion battery) in order to improve the applicability in portable devices and electric vehicles [1].

Lithium-ion batteries are the most important energy storage systems and their total global market is $\sim 29.86$ billion dollars with growing at a Compound Annual Growth Rate (CAGR) of 18.7\% in the next years [2].

This growth results from the growth of the electric vehicle market and the growth of smart devices. Thus, it is necessary a continuous development to improve the properties and characteristics of the different battery components: electrodes and separators [3]. The electrodes are denominated as anode (negative electrode) and cathode (positive electrode) and determine the cell voltage and the battery capacity, respectively [3].

The separator is localized between the two electrodes and serves as the medium for the transfer of the lithium ions during charge and discharge process. Typically, this structure is a porous polymer membrane with electrolyte solution (lithium salts dissolved in organic solvents) [4].

A strategy to increase battery performance is the use of three-dimensional (3-D) batteries in which their performance increases due to the result of the largest ratio of electrode surface area to volume and the lower ohmic losses [5]. In this case, the separator is a solid element with electrolyte solution. The effect of different geometries

International Conference of Numerical Analysis and Applied Mathematics ICNAAM 2019

AIP Conf. Proc. 2293, 260006-1-260006-5; https://doi.org/10.1063/5.0026453

Published by AIP Publishing. 978-0-7354-4025-8/\$30.00 
on the battery separator has been poorly studied and recently it has been demonstrated that different geometries have an impact in increasing cycling behavior of lithium-ion batteries [6].

Thus, the present study focuses on the theoretical optimization of a novel cone structure for battery separators as well as to study its thermal behavior, as shown in figure 1. This cone structure is very interesting because it can control the discharge value taking into account the radius size on one side keeping the other size constant.

\section{METHODOLOGY}

In this work, battery performance and the ohmic heat produced in the bulk separator when radius 1 increase from $0.005 \mathrm{~mm}$ to $0.038 \mathrm{~mm}$ is evaluated. The theoretical simulation was carried out by the Doyle/Fuller/Newman model and the Finite Element Method through the various equations describing the separator and cathode during battery operation.

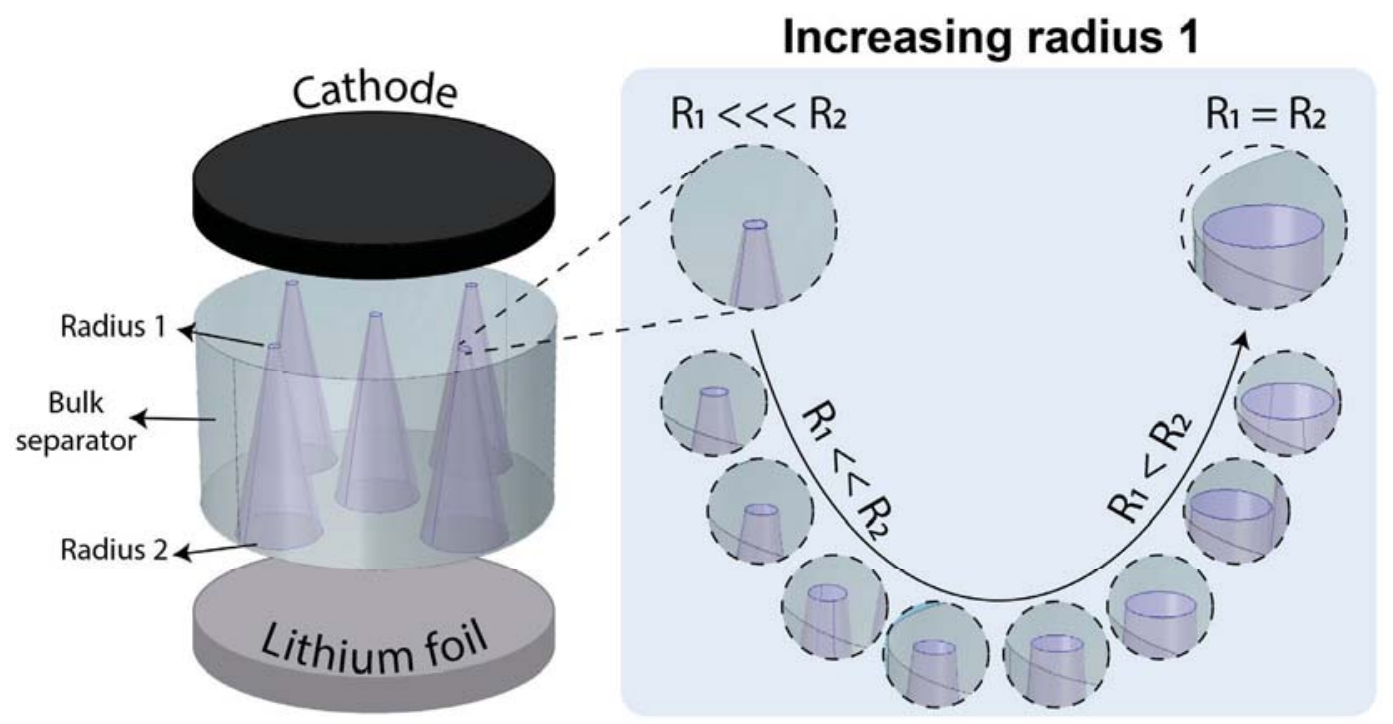

FIGURE 1. Schematic representation of the cone porous separator geometry with different upper base cone radius values applied in the simulated batteries.

Table 1 presents the main values of the different parameters used in the simulations of the separator and the cathode in $\mathrm{Li} / \mathrm{Li}_{x} \mathrm{FePO}_{4}$ half cells. The complete mathematical formulation of the simulations can be found in [7].

TABLE 1. Values of the different parameters used in the developed simulations.

\begin{tabular}{|c|c|c|c|}
\hline \multicolumn{4}{|c|}{ Electrochemical parameters of the components } \\
\hline Parameter & Unit & Separator & Cathode $\left(\mathrm{Li}_{x} \mathrm{FePO}\right)_{4}$ \\
\hline$C_{E, i, 0}$ & mol.m $\mathrm{m}^{-3}$ & & 3900 \\
\hline$C_{E, i, \max }$ & mol.m $\mathrm{m}^{-3}$ & & 21190 \\
\hline$C_{L}$ & mol.m-3 & 1000 & \\
\hline$L_{i}$ & $\mathrm{~m}$ & $150 \times 10^{-6}$ & $20 \times 10^{-6}$ \\
\hline$k_{i}(T)$ & S.m $\mathrm{m}^{-1}$ & a) & a) \\
\hline$D_{i}(T)$ & $\mathrm{m}^{2} \cdot \mathrm{s}^{-1}$ & b) & b) \\
\hline$D_{e f, i}$ & $\mathrm{~m}^{2} \cdot \mathrm{s}^{-1}$ & $D_{i}(T) \times 4.84 \times 10^{-2}$ & $D_{i}(T) \times 0.444^{1,5}$ \\
\hline$D_{L I}$ & $\mathrm{~m}^{2} \cdot \mathrm{s}^{-1}$ & & $3.2 \times 10^{-13}$ \\
\hline$D_{L I}(T)$ & & & c) \\
\hline$\varepsilon_{i}$ & & 0.70 & 0.444 \\
\hline$\sigma_{i}$ & S.m ${ }^{-1}$ & & 11.8 \\
\hline$i_{1 C}$ & A. $m^{-2}$ & 17.5 & \\
\hline$E_{a d, i}$ & J.mol ${ }^{-1}$ & & $39 \times 10^{3}$ \\
\hline$E_{a k, i}$ & J.mol ${ }^{-1}$ & & $29 \times 10^{3}$ \\
\hline$T$ & $\mathrm{~K}$ & 298.15 & 298.15 \\
\hline
\end{tabular}


a) Model Fits: Ionic conductivity as a function of salt concentration in the $\mathrm{LiPF}_{6}$ in EC:DMC mixture [7]: $k i(T)=c \times\left(-10.5+(0.0740 \times T)-\left(\left(6.9610^{-5}\right) \times\left(T^{2}\right)\right)+(0.668 \times c)-(0.0178 \times c \times T)\right.$ $\left.+\left(\left(2.8 \times 10^{-5}\right) \times c \times\left(T^{2}\right)\right)+\left(0.4949 \times c^{2}\right)-\left(\left(8.86 \times 10^{-4}\right) \times\left(c^{2}\right) \times\left(T^{2}\right)\right)\right)^{2}$

b) Diffusion coeficiente as a function of temperature [7]

$D_{i}(T)=10^{\left(-(0.22 c)-4.43-\left(\left(\frac{54}{T-229-5 c}\right)\right)\right)}$

c) Diffusion cefficient of Li ions [7]

$D_{L i}(T)=D_{t 298,15 i} e^{-E_{a d, i} / R}\left(\frac{1}{T}-\frac{1}{298.15}\right)$

\section{RESULTS AND DISCUSSION}

Batteries with cone separator geometry represent a porous separator consisting on a set of cones being filled with electrolyte, as shown in figure 1. For all geometries (increasing radius 1), the volume of the battery components are constant. In this work, radius 1 is increased and therefore, the interface between the separator and the cathode and electrolyte volume also increase. Further, the simulations are performed at different discharge rates (1 and $60 \mathrm{C})$.

Figure 2 shows the different geometries with increasing radius 1 when subjected to the $1 \mathrm{C}$ discharge rate. For this discharge rate, all batteries show the same discharge capacity value, about $167 \mathrm{mAh}^{-\mathrm{g}^{-1}}$. The discharge capacity value does not differ between different samples, as they have been subjected to low discharge rates with easy mobility and higher transport of lithium ions when crossing the separator.

In contrast, at high discharge rates $(60 \mathrm{C})$, an increase in discharge capacity is observed as the radius of the upper base of the cones increases. For the smallest and largest radius (figure 3), the discharge capacity values are 75 $\mathrm{mAh} . \mathrm{g}^{-1}$ and $145 \mathrm{mAh} \cdot \mathrm{g}^{-1}$, respectively, representing an increase of about $97 \%$.

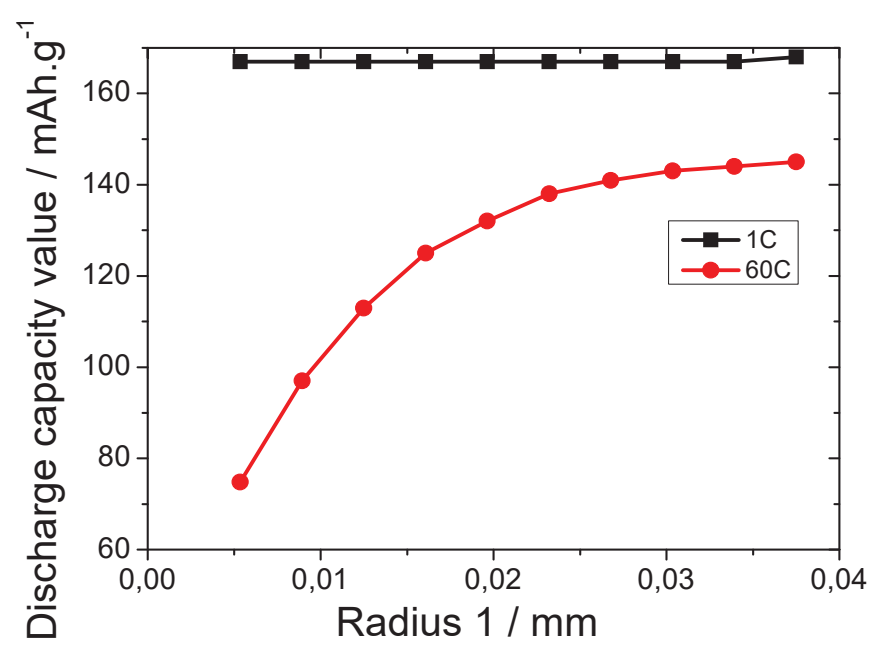

FIGURE 2. Delivered discharge capacity as a function of increasing cone radius 1 for the different samples at $1 \mathrm{C}$ and $60 \mathrm{C}$ scan rate.

Thus, the variation of the geometric parameters of the battery separator significantly influences the discharge capacity values of the batteries when subjected to high discharge rates, due to the diffusion and ion conductivity within the separator.

In this way, the ions have the possibility of crossing long distances through an electrolyte, reducing the path that these ions will have to take in micro-porous bulk separator material during the discharge process. The electrolyte diffusion and ion conductivity values are high compared to the bulk separator diffusion and ion conductivity values. 


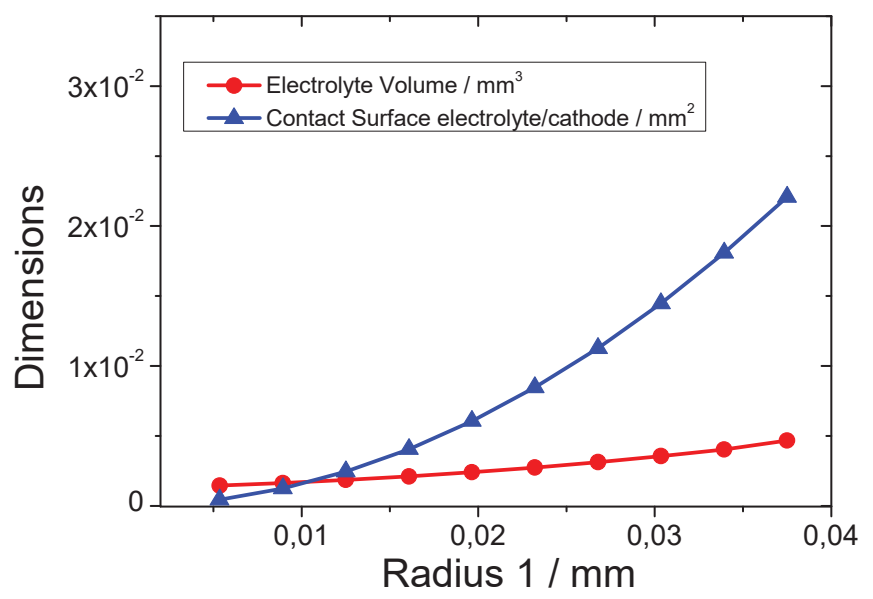

FIGURE 3. Electrolyte volume and contact surface electrolyte/cathode dimensions of the different battery separator samples.

In figure 3 the electrolyte volume increases significantly by $69 \%$ when increasing radius $1(0.005 \mathrm{~mm}$ to 0.038 $\mathrm{mm}$ ). The electrolyte volume values for smallest radius and largest radius are $1.44 \times 10^{-3} \mathrm{~mm}^{3}$ and $4.68 \times 10^{-3} \mathrm{~mm}^{3}$, respectively. Capacity gains depend not only on the volume of electrolyte in the separator, but also on the interface area between the free electrolyte regions and the cathode as is observed in figure 3 . The larger the contact area of the free electrolyte / cathode, the easier it will be for ions to be inserted into the cathode region for later cathode intercalation during the discharge process. In figure 4a), it can be seen that the density of the ionic current in the charge separator regions decreases with increasing radius of the upper base of the cones. The ion density values of the volume current separator from smallest radius to largest radius decrease from $52 \mathrm{~A} . \mathrm{m}^{-2}$ to $17 \mathrm{~A} . \mathrm{m}^{-2}$. As the radius of the upper base of the cones increases, the electrolyte volume increases allowing an increase in ion density in the electrolyte regions and a deficit of their presence in the volume regions of the separators. This fact is also confirmed by the heat produced by the ohmic resistance of the ions in the bulk-separator regions (figure 4b).
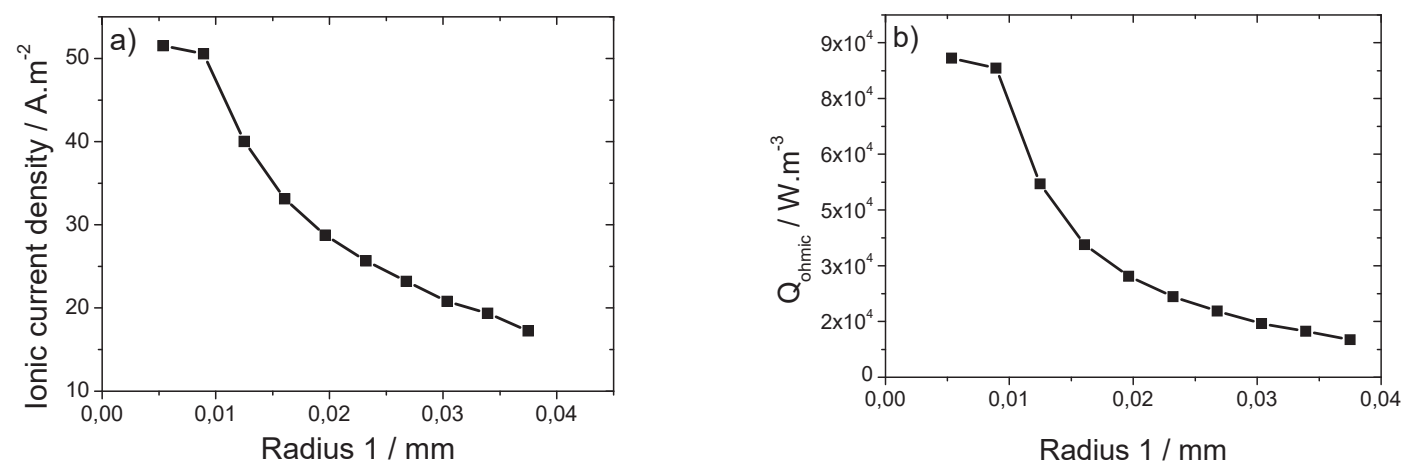

FIGURE 4. a) Ionic current density in bulk separator and b) Ohmic heat produced in bulk separator for increasing radius 1.

The geometry with the smallest radius shows a higher value of produced heat compared to the geometry with the largest radius, the heat produced being $8.6 \times 10^{4} \mathrm{~W} \cdot \mathrm{m}^{-3}$ and $1.01 \times 10^{4} \mathrm{~W} \cdot \mathrm{m}^{-3}$, respectively. This fact is due to the increase of the electrolyte volume.

With the increase in the radius of the upper bases of the cones, there is an increase in electrolyte volume in the separator facilitating the mobility of ions, which in turn increases their presence in this medium, being important to increase the contact area between the electrolyte and the cathode in order to facilitate diffusion and conductivity in these regions, leading to an easier insertion of these into the cathode in the discharge process. 


\section{CONCLUSION}

Optimizing a separator cone structure based on the increasing radius on one side is essential to maximize the performance of the lithium-ion battery. Thus, this work reports on the optimization of this geometry by theoretical simulation based on Doyle/Fuller/Newman model. It was demonstrated that when the radius increases, it allows achieving the best battery performances that depend on the electrolyte volume and interface between free electrolyte/cathode, facilitating the mobility of ions. Also, increasing radius lead to a decrease in the heat produced during the charge/discharge process, preventing the degradation of the battery components. Thus, it was observed that the cone geometry is very interesting for using in the next generation of lithium-ion battery applications due the high capacity discharge at high scan rate.

\section{ACKNOWLEDGMENTS}

Portuguese Foundation for Science and Technology (FCT): UID/FIS/04650/2019, PTDC/CTM-ENE/5387/2014, UID/CTM/50025/2013, PTDC/FIS-MAC/28157/2017, grant SFRH/BPD/112547/2015 (C.M.C.) and contract CEECIND/00833/2017 (R.G.), including FEDER funds through the COMPETE 2020 Programme and National Funds through FCT. Financial support from the Basque Government Industry Department under the ELKARTEK and HAZITEK programs is also acknowledged. F. Miranda was supported by the Portuguese Foundation for Science

and Technology (FCT - Fundação para a Ciência e a Tecnologia), through CIDMA - Center for Research and Development in Mathematics and Applications, within project UID/MAT/04106/2019.

\section{REFERENCES}

1. R. A. Kerr and R. F. Service, Science 309 (2005), 101.

2. Stratistics Market Research Consulting Pvt Ltd, Lithium-ion Battery - Global Market Outlook (2017-2026).

3. B. Scrosati, et al., Journal of Power Sources 195 (2010) 2419-2430.

4. C. M. Costa, et al., Energy storage materials, In press (2019).

5. H. Ragones, et al., Sustainable Energy \& Fuels, 2 (2018) 1542-1549.

6. R. Gonçalves, et al., Energy storage materials, In press (2019).

7. M. Doyle, et al., J. Electrochem. Soc. 143 (1996) 1890-1903. 